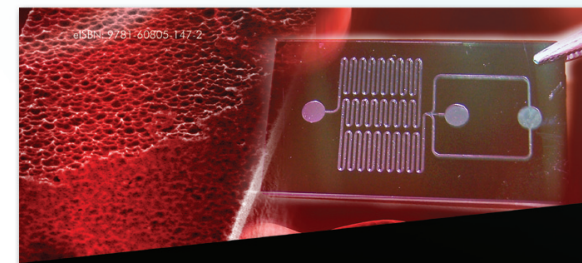

Single user / Non-Library usage

US\$ 24.00

Print-On-Demand (P.O.D)

USS $\mathbf{5 9 . 0 0}$

On-Chip Pretreatment of Whole Blood by Using MEMS

Technology

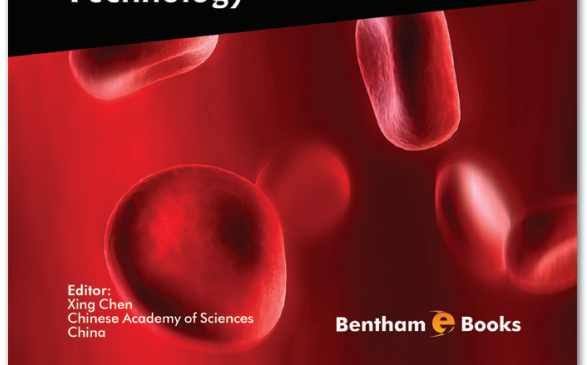

\title{
Editor:
}

Xing Chen

China

\section{Indexed in:}

Scopus

elSBN: 978-1-60805-147-2

\section{On-Chip Pretreatment of Whole Blood by using MEMS Technology}

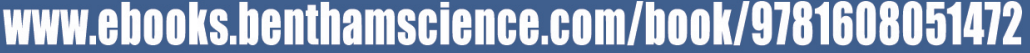

\section{About the eBook}

On-chip pretreatment of whole blood is one of the hottest topics in lab-on-a-chip research since whole blood has been regarded as the most important clinical sample. This e-book is a valuable reference for readers interested in this specific clinical application of advanced MEMS technology.

\section{Contents}

Introduction of Microfluidic Chips Targeting Whole Blood Pretreatment

Microfluidic Chips for Plasma Isolation from Whole Blood

Microfluidic Chips for Blood Cell Separation

- Microfluidic Devices Targeting Blood Cell Lysis

- Microfluidic Chips for DNA Extraction and Purification

Integrated Microfluidic Chips for Whole Blood Pretreatment

\section{For Advertising Inquiries: Contact: marketing@benthamscience.org}

